\title{
Zugangswege bei Azetabulumfrakturen
}

\author{
Ulrich Stöckle, Benjamin König, Max von Seebach, Norbert Haas
}

\section{Zusammenfassung}

Die Wahl des richtigen operativen Zugangsweges ist bei der Versorgung von Azetabulumfrakturen von entscheidender Bedeutung. Es werden einfache Zugänge, die jeweils nur von ventral oder dorsal Manipulationen erlauben, unterschieden von erweiterten Zugängen, die Einsicht und direkte Reposition von beiden Seiten ermöglichen. Der ventrale, ilioinguinale Zugang wird verwendet für Frakturen mit vornehmlicher Pathologie des vorderen Pfeilers und Zweipfeilerfrakturen. Vorteil ist die Weichteilschonung, beachtet werden müssen die Femoralgefäße und der Verlauf des Leistenkanals. Nachteil ist die fehlende Gelenkeinsicht. Der dorsale Kocher-Langenbeck-Zugang wird für Frakturen mit vorwiegend dorsaler Pathologie angewandt. Vorteil des Zuganges ist die Möglichkeit der Gelenkeinsicht, beachtet werden muss der Verlauf des Nervus ischiadicus. Nachteil ist die erhöhte Rate an heterotopen Ossifikationen bei verbleibenden Muskelkontusionen. Zur Verbesserung der Gelenkeinsicht kann der Kocher-Langenbeck-Zugang durch eine bigastrische Trochanterosteotomie ergänzt werden. Erweiterte Zugänge wie der erweiterte iliofemorale bzw. modifiziert erweiterte iliofemorale Zugang (Maryland) werden nur bei solchen Frakturen eingesetzt, die eine direkte Fraktureinsicht und -manipulation von ventral und dorsal erfordern. Vorteil der erweiterten Zugänge ist die gute Übersicht, beachtet werden muss jedoch die Vaskularität der Fragmente und insbesondere des Os ilium, Nachteil ist der erhöhte Weichteilschaden, der mit einer erhöhten Rate an Wundproblemen einhergeht. Für die Zukunft wegweisend ist eine Reduzierung der operativen Zugänge unter zu Hilfenahme der computerassistierten Chirurgie.

\section{Einleitung}

Zur operativen Versorgung von Azetabulumfrakturen sind viele Zugänge beschrieben worden. Im Folgenden sollen die hauptsächlich verwendeten operativen Zugangswege dargestellt werden. Es werden einfache Zugänge, die jeweils nur von ventral oder dorsal Manipulationen erlauben, unterschieden von erweiterten Zugängen, die Einsicht und direkte Reposition von beiden Seiten ermöglichen. Die Wahl des richtigen operativen Zugangsweges ist bei der Versorgung von Azetabulumfrakturen von entscheidender Bedeutung.

OP-JOURNAL 2003; 19: 161-167

(C) Georg Thieme Verlag Stuttgart · New York
Basierend auf einer korrekten Frakturklassifikation wird entschieden, ob ein ventraler oder ein dorsaler Zugang geeignet ist, eine anatomische Gelenkrekonstruktion zu ermöglichen.

Bei Wahl des richtigen Zugangs ist dies in ca. $80 \%$ der Fälle möglich als Voraussetzung für gute funktionelle Resultate. Nur wenn eine direkte Manipulation von beiden Seiten erforderlich ist, kann ein erweiterter Zugang sinnvoll sein. Die Entscheidung für einen erweiterten Zugang sollte hierbei aufgrund der erhöhten Weichteilschäden sehr sorgfältig abgewogen werden. Bei der Versorgung von Azetabulumfrakturen bis zu 3 Wochen nach Trauma sollte die Inzidenz der erweiterten Zugänge 10\% möglichst nicht überschreiten.

\section{Ventraler Zugang ilioinguinal (Abb. 1)}

Während die meisten anderen anterioren Zugänge zum Hüftgelenk sich nur zur Darstellung des Gelenkes eignen und wenig zur Versorgung von Azetabulumfrakturen, gilt der ilioinguinale Zugang als anteriorer Standardzugang für Azetabulumosteosynthesen. Einzig für sehr kraniale Pfannenrandfrakturen und sehr tiefe vordere Pfeilerfrakturen wird der anteriore Zugang nach Smith-Peterson verwendet. Die erweiterte Pfannenstielinzision nach Stoppa ist geeignet für die Versorgung von „Altersfrakturen“ mit Protrusion des Hüftkopfes in das kleine Becken.

Der ilioinguinale Zugang wurde 1960 von Emile Letournel entwickelt. Er ermöglicht die Darstellung des gesamten vorderen Pfeilers, vom SI-Gelenk bis zur Symphyse.

Eine Besonderheit des Zuganges ist, dass in der Regel das Hüftgelenk nicht eingesehen werden kann und sich die Gelenkrekonstruktion deshalb an der anatomischen Reposition der kortikalen Fragmente orientiert.

Lagerung: in Rückenlage, möglichst auf einem röntgendurchlässigen Tisch. Präoperativ ist die Möglichkeit der Bildwandlereinstellungen für a.p., ala und obturator Aufnahme zu testen. Das Bein der verletzten Seite wird frei beweglich abgedeckt. Eine „Kniebeugeschiene“ kann hilfreich sein. Drei unterschiedlich dicke Gummizügel sind bereitzuhalten.

Der Hautschnitt beginnt am Übergang vom hinteren zum mittleren Drittel des Beckenkamms und führt entlang des Beckenkamms zunächst bis zur Spina iliaca anterior superior, um dann entlang des Leistenbandes zu verlaufen und an einem Punkt etwa $2 \mathrm{~cm}$ kranial der Symphyse zu enden. Nach Durchtrennen der Subkutis werden drei sogenannte „Fenster“ präpariert. Durch abwechselnde Retraktion der Weichteile wird so der 

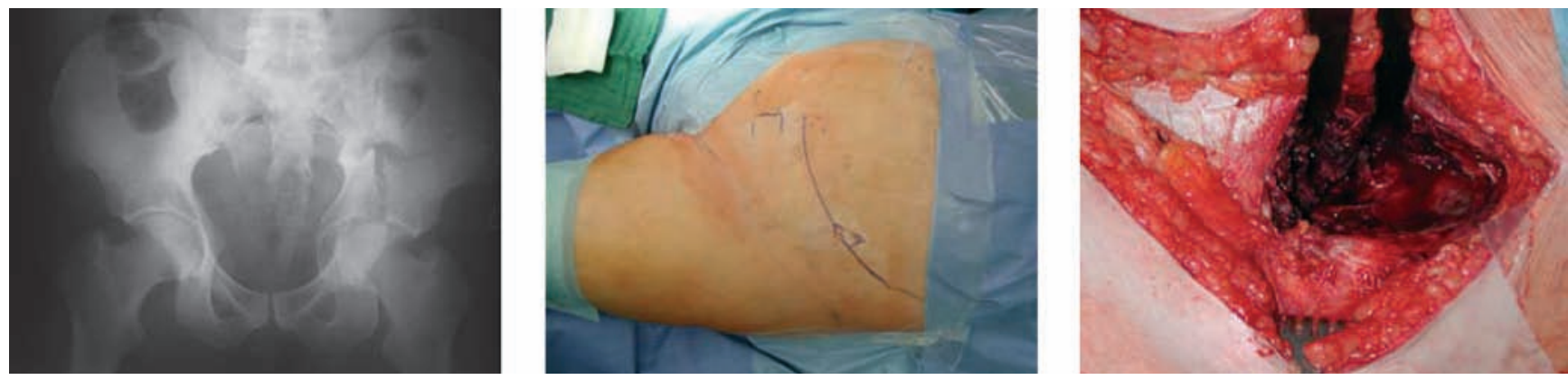

Abb.1a 26-jähriger Pt. mit Azetabulumzweipfeilerfraktur links, bis in das SI-Gelenk auslaufend. Versorgung über ilioinguinalen Zugang. Präoperatives Röntgenbild, Markieren der Hautinzision, Präparation des ersten Fensters über den Beckenkamm.
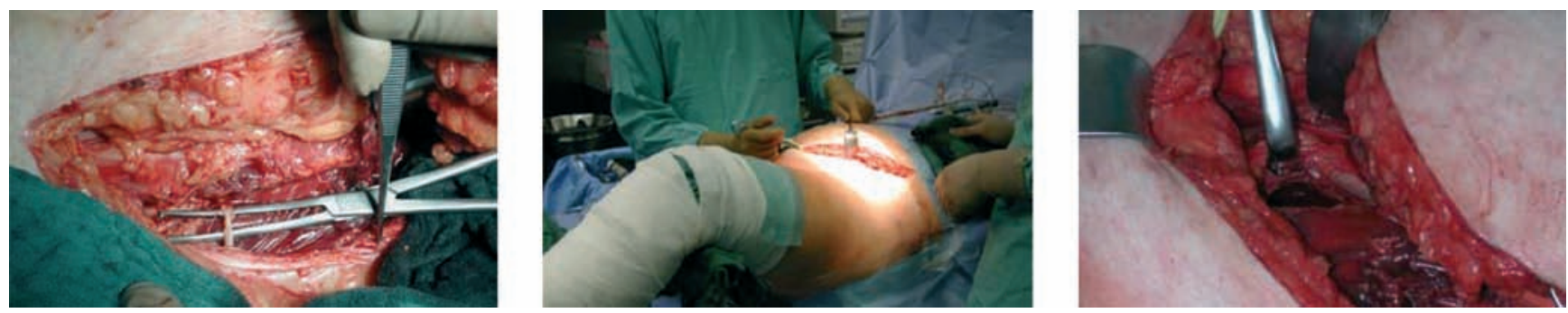

Abb.1b Darstellen des N. cutaneus femoris lateralis, der in diesem Fall sehr ungewöhnlich ca. $5 \mathrm{~cm}$ unterhalb der Spina (Pinzette) verläuft. Einsatz der Kniebeugeschiene zur Entspannung der Hüftbeugemuskulatur während der Präparation. Nach Anschlingen des M. iliopsoas, Darstellen und Inzision des Arcus iliopectineus.
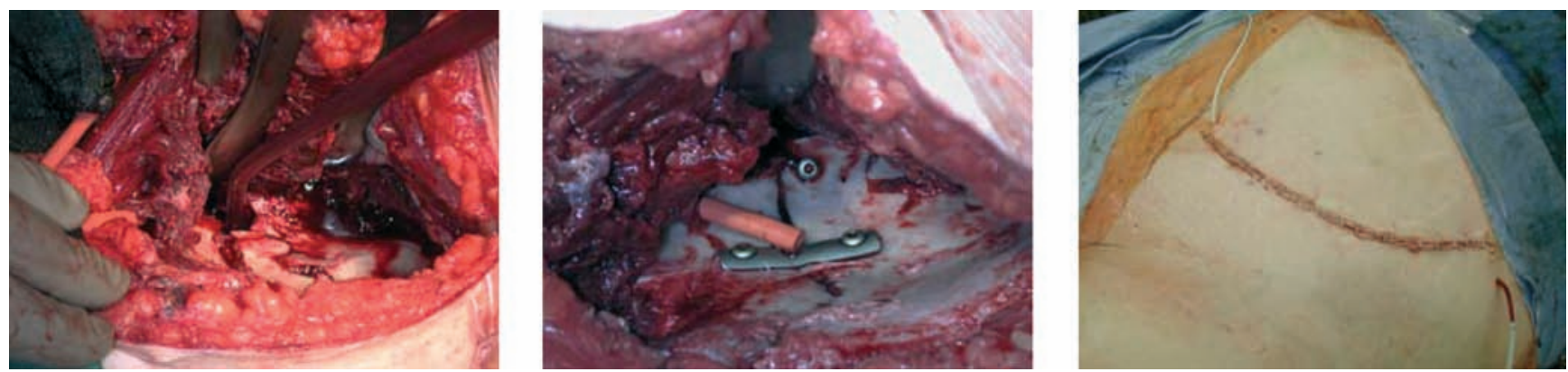

Abb.1c Sukzessive Präparation der Fraktur. Osteosynthese mit Kleinfragmentzugschrauben und Platten. Situation nach Hautverschluss.

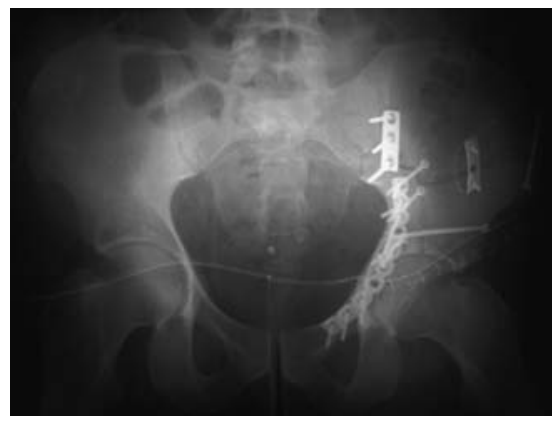

Abb.1d Postoperatives Röntgenbild.

gesamte vordere Pfeiler zugänglich, über das zweite Fenster auch die ganze quadrilaterale Fläche.

Das erste Fenster verläuft entlang der Beckenschaufelinnenseite. Nach dem scharfen Ablösen der Bauchwandmuskulatur vom Beckenkamm, wird mit dem gebogenen Raspatorium subperiostal die Beckenschaufelinnenseite bis in die Fossa iliaca präpariert. Ein runder Hohmann-Haken kann an der Linea terminalis eingesetzt werden. Blutungen aus den Foramina nutricia der Beckenschaufel werden mit Knochenwachs gestillt und die gesamte Wunde vorübergehend mit Rollen oder Bauchtüchern tamponiert.

Zur Darstellung des zweiten Fensters wird nach Durchtrennen der Subkutis zunächst die Aponeurose des M.obliquus externus eröffnet, wobei der äußere Leistenring erhalten werden sollte. Die Ränder der Aponeurose werden mit scharfen Klemmen gefasst, der Leistenkanal eröffnet und die Weichteile mit kleinen Stieltupfern abpräpariert. Der Samenstrang bzw. das Lig.teres uteri werden aufgesucht, einschließlich des N.ilioinguinalis mit dem Finger unter- fahren und schließlich mit einem weichen Zügel angeschlungen. Anschließend wird die Rückwand des Leistenkanals eröffnet, indem der gemeinsame Ursprung des M. obliquus internus, des M. transversus abdominis und der Faszia transversalis vom Leistenband scharf abgelöst werden, so dass ca. $1 \mathrm{~mm}$ des Leistenbandes zur späteren Refixation am Muskel verbleibt. Durch schrittweise Präparation nach lateral wird der N. cutaneus femoris lateralis dargestellt, der sehr variabel in seiner Lage zur Spina iliaca anterior superior verlaufen kann. Eine Durchtrennung hat den Ausfall der Sensibilität an der Oberschenkelaußenseite zur Folge. Bei der Präparation nach medial in Richtung der Lacuna vasorum, wird der Arcus iliopectineus dargestellt, der die Gefäßloge von der Muskelloge trennt. Nach Sicherung der Gefäße mit einem schmalen Langenbeck Haken sowie des M. iliopsoas 
und N. femoralis wird der Arcus iliopectineus wie ein Segel aufgespannt und bis zu seinem Ansatz mit der Schere inzidiert. Nach Ablösen des hinteren Faszienblattes des M.iliopsoas ist so der Zugang zum kleinen Becken frei. Bei gebeugtem Hüftgelenk wird der M.iliopsoas von lateral und medial unterfahren und mit einem kräftigen Zügel angeschlungen. Das erste Fenster im Bereich der Beckenschaufel und das zweite zwischen M.iliopsoas und den Femoralgefäßen ist nun frei zugänglich. Hierüber können die gesamte quadrilaterale Fläche und der Großteil des vorderen Pfeilers digital kontrolliert werden.

Das dritte Fenster liegt zwischen den Femoralgefäßen, die so wenig wie möglich präpariert werden und mit einem mittleren Gummizügel angeschlungen werden, und dem Samenstrang beim Mann bzw. dem ligamentum rotundum bei der Frau. Bei der Präparation entlang des oberen Schambeinastes ist darauf zu achten, ob eine Anastomose zwischen der A. iliaca externa und der A. obturatoria im Sinne einer Corona mortis erweitert ist, die dann zuerst legiert werden sollte. Ist nur eine symphysennahe Fixion der
Platte erforderlich, reicht es meist aus den $\mathrm{M}$. rectus abdominis von lateral mit einem Haken zu retrahieren.

Die einzelnen Fenster werden abwechselnd präpariert und so die Fraktur sukzessive dargestellt. Um die Hüftbeugemuskulatur zu entspannen, ist zwischenzeitlich die Lagerung des Beines in Flexion von Hüfte und Knie auf einer Kniebeugeschiene sinnvoll. Über das erste Fenster ist bei Retraktion des M. iliopsoas nach medial die gesamte Beckenschaufelinnenseite vom SI-Gelenk bis zur Eminentia iliopectinea einsehbar. Das zweite Fenster erlaubt die Einsicht und Palpation entlang der Linea terminalis bis zur Mitte des Foramen obturatum, sowie der gesamten quadrilateralen Fläche, Spina ischiadica und Incisura ischiadica major. Das dritte Fenster erlaubt die Darstellung des oberen Schambeinastes.

Da bei dem ventralen Zugang in der Regel keine Einsicht in das Gelenk möglich ist, ist die exakte anatomische Rekonstruktion der Kortikalisoberfläche entscheidend für eine gute Gelenkrekonstruktion. Die temporäre Fixation wird mit 1-6-mm- oder 2-0-mm, K-Drähten bzw. den Becken-Repositionszangen erreicht. Die Stabilisierung erfolgt zumeist mit Kleinfragment Zugschrauben und einer gebogenen 10-12-Loch-Kleinfragment-Rekonstruktionsplatte, die entlang der Beckeneingangsebene aufgelegt wird. Für Frakturen, die in die Beckenschaufel auslaufen, können weitere Kleinfragmentplatten verwendet werden.

Der Wundverschluss beginnt mit der Rekonstruktion des Leistenkanals. Mit fortlaufender Naht (PDS 0) werden der M. obliquus internus und der M. transversus abdominis an den Unterrand des Leistenbandes refixiert. Hierbei ist beim Mann auf die Durchgängigkeit des inneren Leistenringes $\mathrm{zu}$ achten. Anschließend wird die Aponeurose des M. obliquus externus mit fortlaufender Naht verschlossen (Vicryl 2/0), wobei der Verlauf des N. ilioinguinalis und des N.cutaneus femoralis lateralis beachtet werden müssen. Nach Einlage der tiefen Redondrainage wird dann die Abdominalmuskulatur im Bereich des Beckenkammes refixiert (Vicryl 1/0). Schließlich erfolgt nach Einbringen der Subkutandrainage der Subkutan- und Hautverschluss.
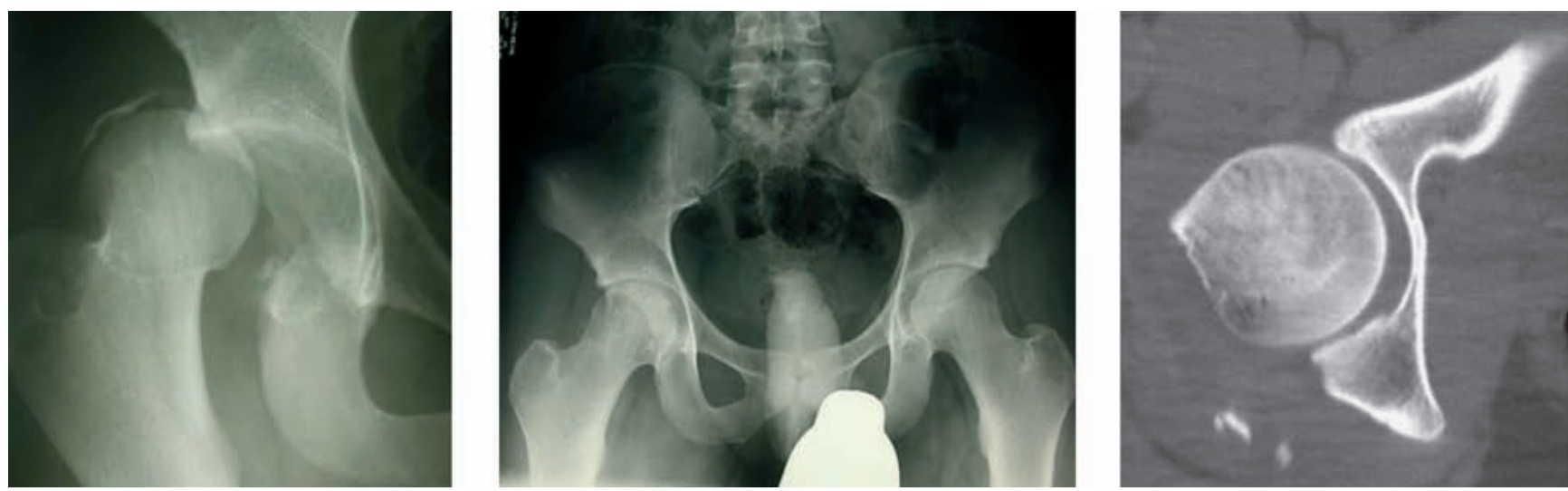

Abb. 2a 21jähriger Pt. mit hinterer Hüftluxation und mehrfragmentärer hinterer Wandfraktur. Luxationsbild, Beckenübersicht nach Reposition, präoperatives CT.
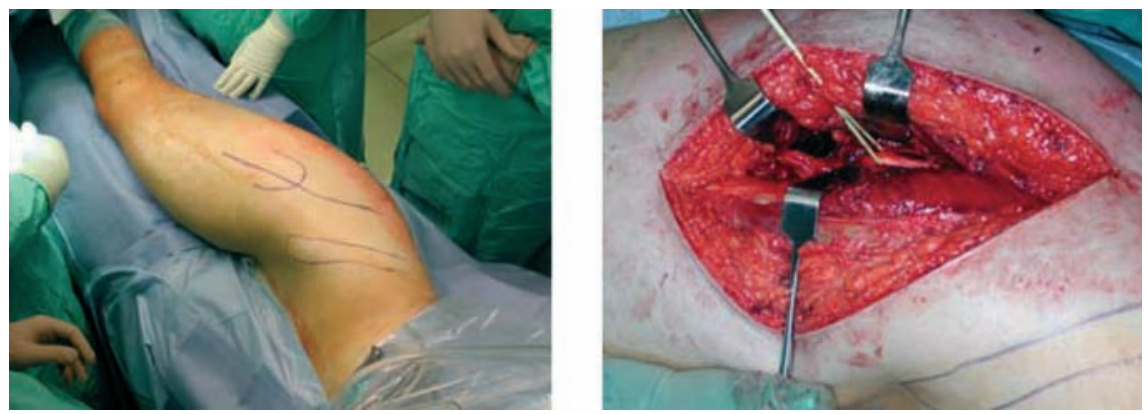

Abb. 2b Lagerung in Seitenlage, das Bein der verletzten Seite frei beweglich abgedeckt. Markieren der Hautinzision. Nach Durchtrennen von Haut, Subkutis und Traktus, Darstellen und Anschlingen des N. ischiadicus. Sicht jeweils von oben. 

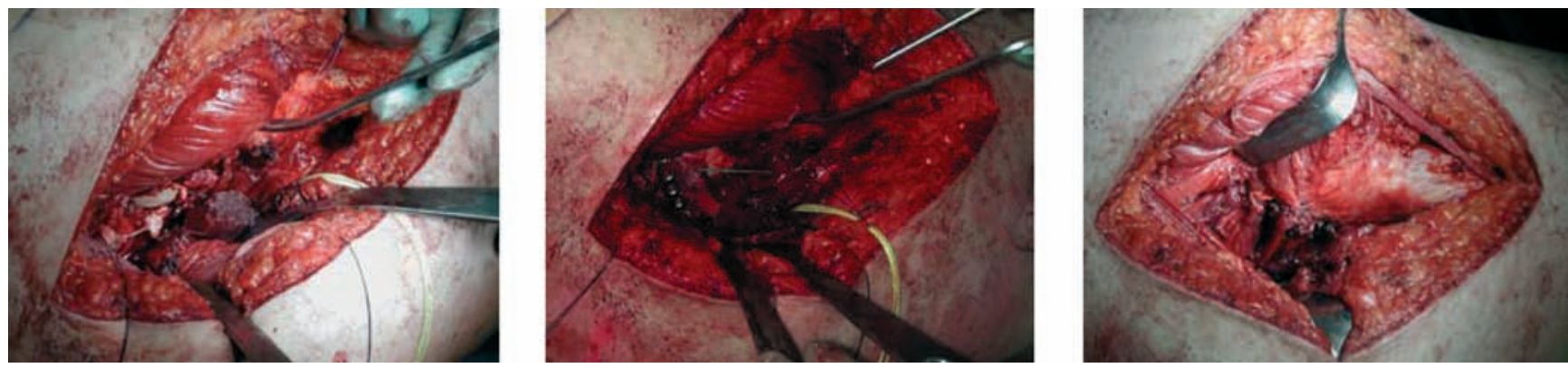

Abb. 2c Sicht von dorsal (Sicht des Operateurs). Darstellen der Fraktur nach Ablösen der Außenrotatoren. Situation nach Fixation der Fragmente mit K-Drähten und Anlage der Platte. Schanz-Schraube im Trochanter major, Hohmann Haken unter dem M. gluteus medius. Refixation der Außenrotatoren.

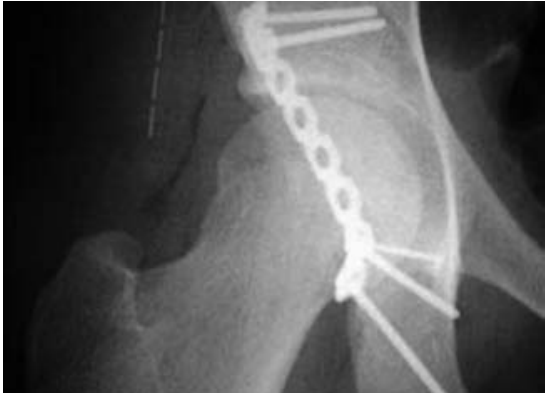

Abb.2d Postoperatives Röntgenbild.

Erreichbarkeit: Durch den ilioinguinalen Zugang kann die gesamte Beckenschaufelinnenseite bis zum SI-Gelenk eingesehen und palpiert werden, der ganze vordere Pfeiler und die quadrilaterale Fläche bis zu Spina ischiadica. Der hintere Pfeiler kann nur im Bereich der Incisura ischiadica major getastet werden. Die Einsicht in das Gelenk ist in der Regel nicht möglich.

Indikationen: Frakturen, die nur den vorderen Pfeiler betreffen (vordere Wand, vorderer Pfeiler); Querfrakturen mit Versatz nach ventral; kombinierte Frakturtypen mit der Hauptpathologie ventral und nicht oder nur minimal dislozierten Frakturkomponenten des hinteren Pfei-

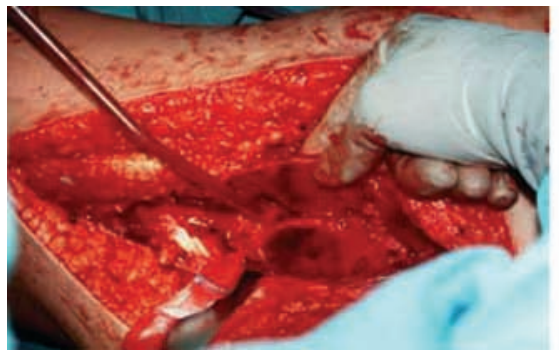

lers; Zweipfeilerfrakturen, die von ventral rekonstruiert werden können.

Vorteile: Bei Kenntnis der Anatomie weichteilschonender Eingriff mit wenig Wundheilungsproblemen und nahezu keinen heterotopen Ossifikationen.

Nachteile: Keine direkte Gelenkeinsicht.

Risiken: Verletzung des N.cutaneus femoris lateralis $(23 \%$ in der Serie von Letournel), Verletzung der Femoralgefäße, Lymphödem des betroffenen Beines nach Gefäßskelettierung,

Erweiterungsmöglichkeiten: Zum Einsatz großer Repositionszangen kann der Ansatz der Abduktoren im Bereich der Crista iliaca eingekerbt werden. Um einen Zugang auf die Rückseite des Iliums zu gewinnen, ist auch die zusätzliche Osteotomie der Spina iliaca anterior superior bzw. Ablösung der Sehne des M. rectus femoris als Erweiterung beschrieben worden.

\section{Dorsaler Zugang Kocher-Langenbeck (Abb. 2)}

Der hintere Zugang ist der am häufigsten verwendete Zugang zur operativen Versorgung von Azetabulumfrakturen.
Der Kocher-Langenbeck-Zugang bietet eine sehr gute Übersicht über die Außenseite des Beckens und ermöglicht zumindest palpatorisch eine ausreichende Kontrolle einfacher Frakturlinien des vorderen Pfeilers.

Die Lagerung erfolgt entweder in Bauchlage oder in Seitenlage. Auch wenn in Bauchlage eine weitergehende Präparation des Zuganges möglich ist, wird im eigenen Vorgehen die Seitenlage bevorzugt aufgrund der besseren Manipulierbarkeit des frei beweglich abgedeckten Beines. Bei der Abdeckung muss darauf geachtet werden, dass die Spina iliaca posterior tastbar ist. Während der gesamten Operation ist das Knie gebeugt zu halten, um so jegliche Spannung auf den $\mathrm{N}$. ischiadicus zu vermeiden.

Die Hautinzision verläuft zunächst entlang der Femurachse und biegt dann in Höhe des Trochanter major in Richtung Spina iliaca posterior superior ab bis ca. $6 \mathrm{~cm}$ vor der Spina. Nach Durchtrennen der Subkutis wird die Faszia lata in ihrem dorsalen Anteil inzidiert und der M. gluteus maximus in Faserrichtung gespalten. Dies geschieht am besten stumpf mit dem Finger, um Läsionen der superioren glutealen Nerven und der Gefäße zu vermeiden. Die meist eingeblutete Bursa tro-

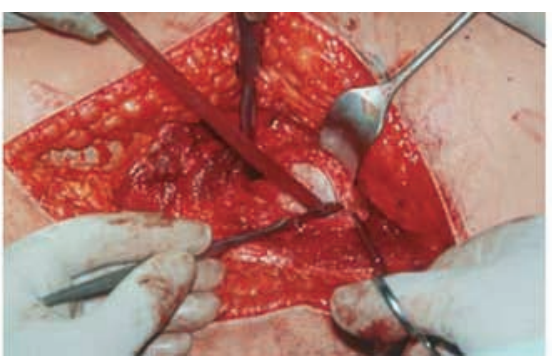

Abb. 3 Erweiterung des dorsalen Zuganges mit bigastrischer Trochanterosteotomie (Trochantermedaillon mit anhängendem M. gluteus medius und M. vastus lateralis). Nach Präparation des Medaillons nach ventral, Möglichkeit der zirkulären Kapsulotomie und Luxation des Hüftkopfes. Auf diese Weise gute Einsicht in das Gelenk. 


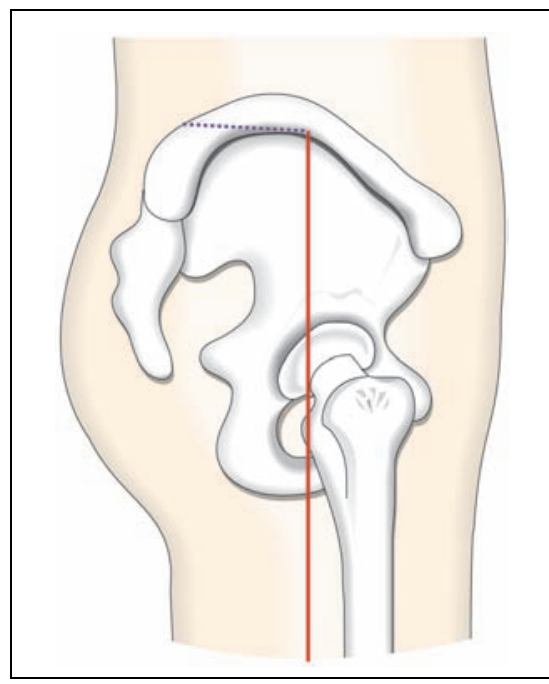

Abb. 4a Modifiziert erweiterter iliofemoraler Zugang (Maryland). T-förmige Hautinzision. Präparation erst des dorsalen Zugangs. Dann Ablösen des M. tensor fasziae latae.

chanterica wird exzidiert und der Tractus iliotibialis nach distal gespalten, bis in Höhe der Linea aspera, dem Ansatz des M. gluteus maximus. Der Nervus ischiadicus wird aufgesucht, am leichtesten im Bereich des zumeist unverletzten M.quadratus femoris. Nach Darstellung des Verlaufes und Anschlingen des Nerven wird zunächst der M.piriformis, der an seiner starken Sehne und seinem Verlauf auf dem Nerven zumeist gut identifizierbar ist, ca. $1 \mathrm{~cm}$ von seinem Ansatz abgesetzt und mit Haltefäden markiert. Ebenso werden dann die Außenrotatoren (M.obturator internus, mm. gemelli) vom Ansatz abgelöst und mit Haltefäden versehen. Die am Oberrand des M. quadratus verlaufende Arteria circumflexa femoris profunda ist unbedingt zu schonen, da sie entscheidend ist für die Durchblutung des Hüftkopfes. Nach Einsetzen eines runden Hohmann-Hakens in die Bursa obturatoria, der die Außenrotatoren unter Schutz des N. ischiadicus retrahiert und eines schmal, spitzen Hohmann-Hakens zur Retraktion der Abduktoren erfolgt dann sukzessive die Darstellung der Fraktur. Die Kapsel wird azetabulumnah inzidiert, falls die traumatischen Kapseleinrisse zur Exposition nicht ausreichen. Die Einsicht in das Gelenk kann hierbei durch Extraktion des Hüftkopfes mit einer vom Trochanter in den Schenkelhals eingebrachten SchanzSchraube verbessert werden. Zur Osteosynthese wird neben KleinfragmentZugschrauben meist eine vorgebogene 8- oder 10-Loch-Kleinfragment-Rekonstruktionsplatte an die Kontur der Azeta-
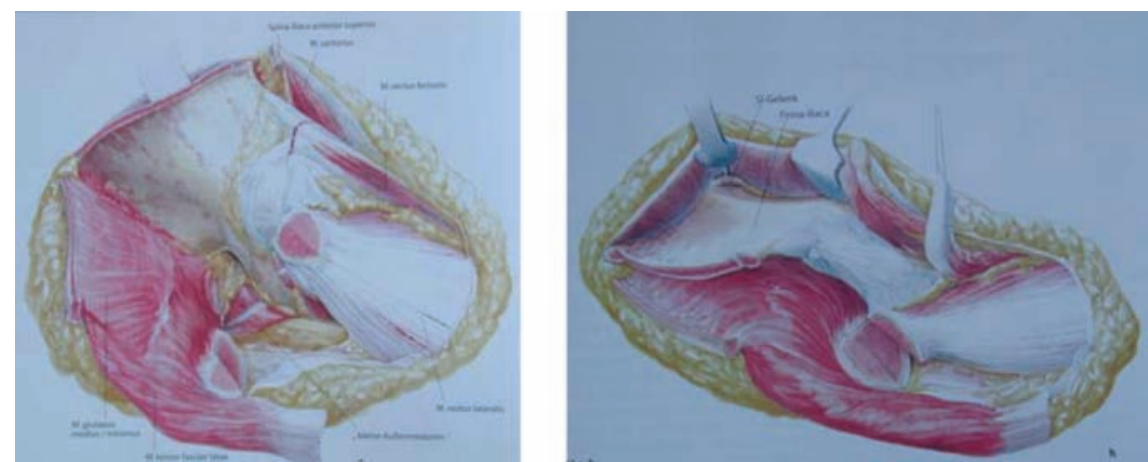

Abb. 4b Exposition der gesamten Außenseite der Beckenschaufel nach Trochanter-Osteotomie und Osteotomie des Beckenkamms. Nach Osteotomie der Spina iliaca anterior superior und scharfem Ablösen des M. rectus ist auch die Innenseite der Beckenschaufel zugänglich bis zum Pecten ossis pubis (Abbildungen aus: Tscherne/Pohlemann: Unfallchirurgie, Becken/Azetabulum).
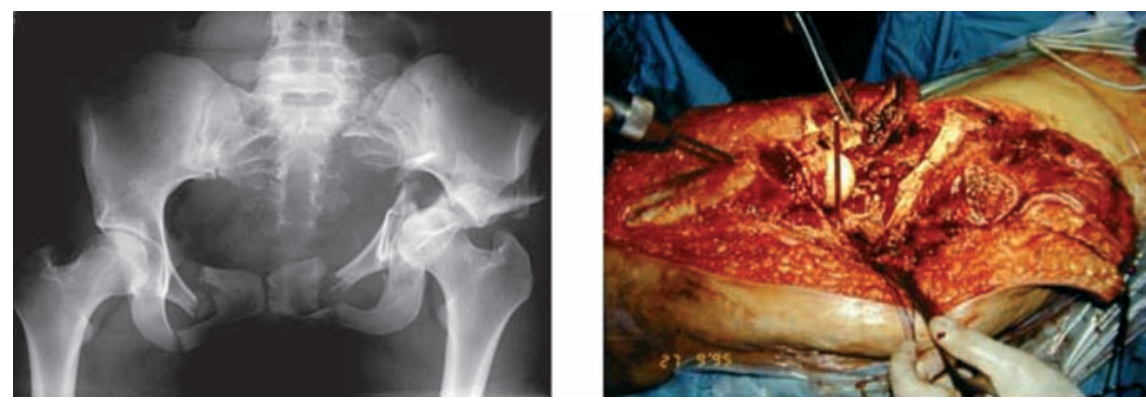

Abb.4c Röntgen-Beckenübersicht einer 18-jährigen Pt. mit Sakrumfraktur li. und Azetabulumzweipfeilerfraktur links mit erheblicher Trümmerzone. Intraoperative Ansicht bei modifiziert erweitertem iliofemoralen Zugang. Eine Schanz-Schraube ist im Trochanter, um den Hüftkopf führen zu können. Eine weitere ist im Os ischii für die spätere Reposition. Man beachte die große Weichteilexposition.
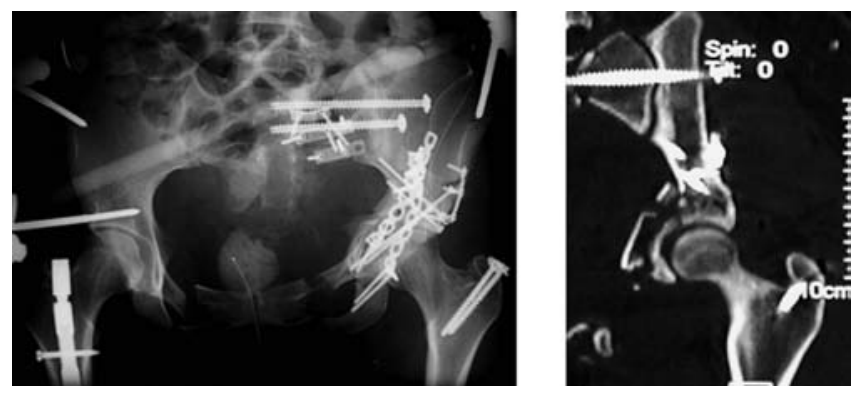

Abb. 4d Postoperative Röntgen- und CT-Kontrolle der Repositionsqualität.

bulumhinterwand angepasst und proximal in die Beckenschaufel, distal in das Os ischium mit mindestens jeweils 2 Schrauben fixiert. Zusätzliche Platten aus dem Kleinfragmentinstrumentarium können zur Erhöhung der Stabilität erforderlich sein. Falls nicht bereits vorher erfolgt, sollten kontusionierte Anteile der Muskulatur reseziert werden, um in der Folge die Bildung von heterotopen Ossifikationen zu vermeiden. Der Wundverschluss beginnt nach ausgiebiger Spülung mit der Refixation der Kapsel (PDS 0). Dann werden der M. piriformis und die Außenrotatoren wieder an ihren Ansatz refixiert (PDS 0), ein tiefes Redon eingelegt, die Faszie genäht (Vicryl 1/0), ein subkutanes Redon eingelegt und schließlich Subkutis und Haut verschlossen.

Erreichbarkeit: Über den Kocher-Langenbeck-Zugang ist die Außenfläche des Os innominatum vom Tuber ischiadicum bis zur Incisura ischiadica major zugänglich. Die Innenseite ist bis nahe an die Linea terminalis palpatorisch erreichbar.

Indikationen: Frakturen der hinteren Wand oder des hinteren Pfeilers; Transtektale und juxtatektale Querfrakturen; Querfrakturen mit Frakturen der hinteren Wand.

Vorteile: Direkte Gelenkeinsicht. 
Nachteile: Erhöhte Rate an heterotope Ossifikationen

Komplikationen: Nervenläsionen (N. ischiadicus, N. gluteus inferior und superior), Außenrotationsschwäche.

\section{Erweiterungsmöglichkeit (Abb. 3):}

Um die Gelenkeinsicht zu verbessern, kann der dorsale Zugang mit einer bigastrischen Trochanterosteotomie erweitert werden.

Hierbei wird nach Darstellung der Ansatzbereiche, mit der oszillierenden Säge ein Trochantermedaillon osteotomiert mit anhängendem $\mathrm{M}$. gluteus medius und M. vastus lateralis. Indem das Trochantermedaillon nach ventral gehalten wird, kann die Gelenkkapsel auch im gesamten ventralen Bereich dargestellt und inzidiert werden. Eine komplette Luxation des Hüftkopfes ist so möglich und erlaubt die direkte Kontrolle der Gelenkrekonstruktion. Die Refixation des Trochantermedaillons erfolgt mit 2 - 3 Kleinfragmentschrauben bzw. kanülierten 4,5-mm-Schrauben.

\section{Erweiterter Zugang (Maryland-Zugang) (Abb. 4)}

Als klassischer erweiterter Zugang zur Versorgung von Azetabulumfrakturen ist der erweiterte iliofemorale Zugang bekannt, wie er von Judet und Letournel 1973 beschrieben wurde. Im eigenen Vorgehen wird jedoch der modifiziert erweiterte iliofemorale Zugang bevorzugt, bei dem, stufenweise erweiterbar, die Muskulatur nicht im sehnigen Bereich abgesetzt wird, sondern zumeist als Osteotomie und somit die Nachbehandlung erleichtert ist. Deshalb wird nachfolgend der sog. „Maryland-Zugang“ beschrieben, wie er von C. Reinert 1988 publiziert wurde.

Lagerung: Seitenlagerung mit der Vakuummatratze. Das Bein der verletzten Seite wird frei beweglich abgedeckt, der gesamte Beckenkamm von der Spina iliaca anterior superior bis zur Spina iliaca posterior superior sollte frei zugänglich sein.

Der Hautschnitt für den modifiziert erweiterten iliofemoralen Zugang verläuft T-förmig. Der quere Schenkel ist ca. $12 \mathrm{~cm}$ lang, an der Spina iliaca anterior superior beginnend entlang des Beckenkammes. Am Übergang vom ventralen zum mittleren Drittel verläuft dann rechtwinklig dazu der ca. $30 \mathrm{~cm}$ lange Längsschenkel, der zunächst bogenförmig zum Trochanter major zieht und dann entlang der Femurachse liegt. Nach Durchtrennen der Subkutis werden zwei subkutane Lappen präpariert. Der Traktus iliotibialis wird inzidiert und zunächst der dorsale Zugang, wie oben beschrieben, entwickelt. Nach Darstellung des Muskelbauches des M.tensor fasziae latae wird dieser U-förmig nach kranial abgelöst, die A.circumflexa femoris lateralis wird hierbei legiert. Die Spina iliaca anterior superior wird osteotomiert und so der M. sartorius knöchern abgelöst. Der M. rectus femoris wird im sehnigen Ansatzbereich einschließlich der in die Kapsel einstrahlenden Pars reflexa scharf abgesetzt.

Schließlich wird der Trochanter osteotomiert, der M. gluteus nach oben geschlagen. Zur Darstellung der Beckenschaufelinnenseite wird die Bauchwandmuskulatur am Beckenkamm scharf abgelöst. Um die komplette Außenseite der Beckenschaufel zugänglich zu machen, wird der Beckenkamm osteotomiert. Im eigenen Vorgehen wird hier eine Schrägosteotomie bevorzugt.

Nach Komplettierung des Zugangs is die gesamte Außenseite der Beckenschaufel direkt zugänglich, die Gelenkeinsicht von dorsal möglich und die Innenseite des Beckens vom SI-Gelenk bis zum Pecten ossis pubis palpierbar.

Es können so von dorsal und ventral die einzelnen Fragmente reponiert werden bei direkter Kontrolle des Gelenkes. Die Osteosynthese erfolgt mit Zugschrauben und Kleinfragmentplatten aus dem Beckeninstrumentarium. Die Osteotomien der Spina iliaca anterior superior und des Beckenkamms werden mit Kleinfragmentzugschrauben bzw. 2,7-mmSchrauben refixiert, der Trochanter mit zwei 6,5-mm-Spongiosaschrauben mit Unterlegscheibe oder zwei kannülierten 7,0-mm-Schrauben. Der M. rectus femoris wird transossär an der Spina iliaca anterior inferior mit PDS-Naht 0 refixiert und die Pars reflexa wieder mit der Gelenkkapsel verbunden. Die Außenrotatoren und der M.piriformis werden ebenso mit PDS 0 wieder an ihren Ansatz fixiert. Schließlich wird der Traktus iliotibialis in Abduktionsstellung des Beines mit Vicryl $1 / 0$ wieder verschlossen.

Mit erweiterten Zugängen ist auch bei komplexen Frakturformen in bis $\mathrm{zu}$ $100 \%$ eine anatomische Rekonstruktion möglich. Im eigenen Vorgehen konnte im Rahmen einer Serie von 50 kombinierten Frakturen, die mit dem Maryland-Zugang versorgt wurden, in 40 Fällen ein anatomisches Repositionsergebnis (verbliebene Stufe $<1 \mathrm{~mm}$ ) erreicht werden. Im 2-Jahres-Follow-up zeigten dann auch 25 von 26 nachuntersuchten Patienten mit initial anatomischem Ergebnis ein gutes bis sehr gutes funktionelles und radiologisches Ergebnis. Bedacht werden muss allerdings die recht hohe Rate an zugangsbedingten Komplikationen wie Hämatoseromen (14\%), heterotopen Ossifikationen (10\%) und Nervenläsionen (4\%) bei einer gesamten Re-Operationsrate von $20 \%$. Im Rahmen der Nachbehandlung sollte eine Ossifikations-Prophylaxe mit Indomethacin oder einer einmaligen Bestrahlung erfolgen.

Die Indikation zu einem erweiterten Zugang sollte deshalb sehr sorgfältig überdacht werden. Nur bei Frakturformen, die sowohl von ventral als auch von dorsal eine direkte Manipulation erfordern, ist in der Primärversorgung die Indikation berechtigt.

Die Inzidenz sollte $10 \%$ nicht übersteigen. Bei Spätrekonstruktionen jedoch bleibt der erweiterte Zugang der Zugang der Wahl für kombinierte Frakturformen.

Erreichbarkeit: Direkte Erreichbarkeit des gesamten hinteren Pfeilers und großer Teile des vorderen Pfeilers auf der Beckenschaufelinnenseite (vom Pecten ossis pubis bis zum SI-Gelenk). Palpatorisch kann die gesamte quadrilaterale Fläche kontrolliert werden.

Indikation: In der Primärversorgung Frakturen mit Frakturpathologie im Bereich beider Pfeiler, die von ventral und dorsal direkt chirurgisch angegangen werden müssen, z.B.: ausgewählten Querfrakturen mit Fraktur der hinteren Wand, T-Frakturen, Zweipfeilerfrakturen mit dislozierten hinteren Wandfrakturen oder Trümmerzonen. Bei der Spätrekonstruktion nahezu alle kombinierten Frakturtypen, wenn beide Pfeiler erreicht werden müssen.

Vorteile: Erweiterte Übersicht im Bereich des hinteren Pfeilers, Darstellung der kompletten Gelenkkapsel.

Nachteile: Ausgedehnte Weichteilexposition und damit verbundene Komplikationen. Für die reine Präparation sind ca. 90 min zu planen. 
Komplikationen: hohe Rate an Hämatoseromen, Nervenschädigung (N. ischiadicus, N.cutaneus femoris lateralis, N. gluteus superior), heterotope Ossifikationen (bis zu 25\% in der Serie von Reinert).

\section{Schlussfolgerung}

Nach korrekter Klassifizierung der Fraktur und Indikationsstellung zur operativen Therapie kommt der Wahl des geeigneten operativen Zugangs entscheidende Bedeutung zu.

Die Wahl eines falschen Zugangs kann dazu führen, dass Teile der Fraktur nicht erreichbar sind und damit eine anatomische Gelenkrekonstruktion nicht möglich ist.

Die simultane Kombination eines dorsalen und ventralen Zuganges hat immer zur Folge, dass zumindest ein Zugang nicht komplett entwickelt werden kann. Der unkritische Einsatz erweiterter $\mathrm{Zu}$ gänge erhöht wiederum das Operationstrauma für den Patienten mit deutlichem Anstieg der perioperativen Morbidität.

Deshalb ist die Kenntnis der Standardzugänge wichtig, um einschätzen $\mathrm{zu}$ können, ob die gesamte Fraktur mit dem jeweiligen Zugang angegangen und damit die Entscheidung für den richtigen Zugang getroffen werden kann. Bei korrekter Zugangswahl kann dann in ca. $80 \%$ der Fälle eine anatomische Gelenkrekonstruktion erreicht werden, die Voraussetzung ist für gut funktionelle Langzeit Resultate. Durch verbesserte Bildgebung und den intraoperativen Einsatz von Navigationsverfahren werden in $\mathrm{Zu}-$ kunft sicherlich auch die bisherigen "Standardzugänge“ in der Ausdehnung und der Inzidenz reduziert werden.

\section{Literatur}

${ }^{1}$ Alonso JE, Davila R, Bradley E. Extended Iliofemoral Versus Triradiate Approaches in Management of Associated Acetabular Fractures. Clin Orthop 1994; 305: 81 - 87

${ }^{2}$ Berton RM, Letournel E. Low-Dose Irradiation and Indomethacin Prevent Heterotopic Ossification after Acetabular Fracture Surgery. J Bone Joint Surg 1994; 76B: 895-900 ${ }^{3}$ Bosse MJ, Poka A, Reinert CM, Ellwanger F, Slawson R, McDevitt ER. Heterotopic Ossification as a Complication of Acetabular Fractures. J Bone Joint Surg 1988; 70A: $1231-1237$

${ }^{4}$ Brooker AF, Bowerman JW, Robinson RA, Riley Jr LH. Ectopic ossification following total hip replacement: Incidence and a method of classification. J Bone Joint Surg 1973; 55A: $1629-32$

${ }^{5}$ Ghalambor N, Matta JM,Bernstein L. Heterotopic Ossification Following Operative Treatment of Acetabular Fracture. Clin Orthop 1994; 305: 96-105

${ }^{6}$ Goulet JA, Rouleau JP, Mason DJ, Goldstein SA, Arbor A. Comminuted Fractures of the Posterior Wall of the Acetabulum. J Bone Joint Surg 1994; 76A: 1457-1463

${ }^{7}$ Heeg M, Klasen HJ, Visser JD. Operative Treatment for Acetabular Fractures. J Bone Joint Surg 1990; 72B: $383-386$

${ }^{8}$ Helfet DL, Schmeling GJ. Management of Complex Acetabular Fractures Through Single Nonextensile Exposures. Clin Orthop 1994; 305: 58-68

${ }^{9}$ Letournel E. Fractures of the acetabulum. In Elson RA (ed). Berlin Springer Verlag 1993

${ }^{10}$ Letournel E. Fractures of the Acetabulum. A Study of a Series of 75 Cases. Clin Orthop 1994; 305: 5-9

${ }^{11}$ Letournel E. The Treatment of Acetabular Fractures Through the Ilioinguinal Approach Clin Orthop 1993; 292: $62-76$

${ }^{12}$ Liebergall M, Mosheiff R, Low J et al. Acetabular fractures. Clinical outcome of surgical treatment Clin Orthop 1999; 366: 205-216

${ }^{13}$ Matta JM. Fractures of the acetabulum: accuracy of reduction and clinical results in patients managed operatively within three weeks after the injury. J Bone Joint Surg Am 1996; 78(11): 1632-1645

${ }^{14}$ Matta JM. Operative Treatment of the Acetabular Fractures Through the Ilioinguinal approach - A Ten Years Perpective. Clin Orthop 1994; 305: 10-19
${ }^{15}$ Matta JM, Anderson LM, Epstein HC, Hendricks P. Fractures of the Acetabulum: A Retrospective Analysis. Clin Orthop 1986; 205: $230-240$

${ }^{16}$ Moed B, Letournel E. Low-dose irradiation and indomethacin prevent heterotopic ossification after acetabular fracture surgery. JBJS [Br] 1994; 76-B: 895-900

${ }^{17}$ Moed B, Karges DE. Prophylactic Indomethacin for the Prevention of Heterotopic Ossification After Acetabular Fracture Surgery in High- Risk Patients. J Orthop Trauma 1994; 8: 34-39

${ }^{18}$ Reinert CM, Bosse MJ, Poka A, Schacherer T, Brumback Robert J, Burgess AR. A Modified Exposure for the Treatment of Complex or Malunited Acetabular Fractures. J Bone Joint Surg 1988; 70A: 329-335

${ }^{19}$ Schmidt CC, Gruen GS. Non-Extensile Surgical Approach for Two- Column Acetabular Fractures. J Bone Joint Surg 1992; 75B: 556-561

${ }^{20}$ Siebenrock KA, Gautier E, Ziran BH, Ganz R. Trochanteric flip osteotomy for cranial extension and muscle protection in acetabular fracture fixation using a Kocher-Langenbeck approach. J Orthop Trauma 1998; 12(6): 387-91

${ }^{21}$ Stannard JP, Alonso JE. Controversies in acetabular fractures. Clin Orthop 1998; 353 . 74-80 Review

${ }^{22}$ Stöckle U, Hoffmann R, Südkamp N, Reindl R, Haas N. Treatment of complex acetabular fractures through a modified extended iliofemoral approach J Orthop Trauma (2002) 16 (4): $220-30$

${ }^{23}$ Tscherne H, Pohlemann T (Hrsg.). Unfallchirurgie, Becken und Acetabulum, 1998

${ }^{24}$ Weber GT, Mast JM. The Extended Ilioinguinal Approach for Specific Both Column Fractures. Clin Orthop 1994; 305: 106-111

Priv.-Doz. med. Dr. Ulrich Stöckle Leitender Oberarzt Dr. med. Benjamin König Wissenschaftlicher Mitarbeiter Dr. med. Max von Seebach Wissenschaftlicher Mitarbeiter Prof. Dr. med. Norbert Haas Ärztlicher Direktor

Unfall- und Wiederherstellungschirurgie Humboldt Universität zu Berlin Charité. Campus Virchow Klinikum Augustenburgerplatz 1 D-13353 Berlin 